\title{
A Paper-Based Invasion Assay: Assessing Chemotaxis of Cancer Cells in Gradients of Oxygen
}

\section{Citation}

Mosadegh, Bobak, Matthew R. Lockett, Kyaw Thu Minn, Karen A. Simon, Karl Gilbert, Shawn Hillier, David Newsome, et al. 2015. "A Paper-Based Invasion Assay: Assessing Chemotaxis of Cancer Cells in Gradients of Oxygen." Biomaterials 52 (June): 262-271. doi:10.1016/ j.biomaterials.2015.02.012.

\section{Published Version}

doi:10.1016/j.biomaterials.2015.02.012

\section{Permanent link}

http://nrs.harvard.edu/urn-3:HUL.InstRepos:25047697

\section{Terms of Use}

This article was downloaded from Harvard University's DASH repository, and is made available under the terms and conditions applicable to Open Access Policy Articles, as set forth at http:// nrs.harvard.edu/urn-3:HUL.InstRepos:dash.current.terms-of-use\#OAP

\section{Share Your Story}

The Harvard community has made this article openly available.

Please share how this access benefits you. Submit a story.

\section{Accessibility}




\title{
A Paper-Based Invasion Assay: Assessing Chemotaxis of Cancer Cells in Gradients of Oxygen
}

\author{
Bobak Mosadegh $^{\mathrm{a}, \mathrm{b}^{\dagger} *}$, Matthew R. Lockett ${ }^{\mathrm{a} \dagger}$, Kyaw Thu Minn ${ }^{\mathrm{a}}$, Karen A. Simon ${ }^{\mathrm{a}}$, Karl Gilbert ${ }^{\mathrm{a}}$, \\ Shawn Hillier ${ }^{\mathrm{c}}$, David Newsome ${ }^{\mathrm{c}}$, Howard Li ${ }^{\mathrm{c}}$, Amy B. Hall' , Diane M. Boucher ${ }^{\mathrm{c}}$, Brenda K. \\ Eustace $^{\mathrm{c} *}$ and George M. Whitesides ${ }^{\mathrm{a}, \mathrm{b}} *$
}

${ }^{a}$ Department of Chemistry and Chemical Biology, Harvard University

12 Oxford Street, Cambridge, MA 02138

${ }^{\mathrm{b}}$ Wyss Institute of Biologically Inspired Engineering, Harvard University

60 Oxford Street, Cambridge, MA 02138

${ }^{\mathrm{c}}$ Vertex Pharmaceuticals Incorporated

50 Northern Avenue Boston, MA 02210

*Authors to whom correspondence should be addressed.

Email: gwhitesides@gmwgroup.harvard.edu

Email: brenda_eustace@vrtx.com

Email: bmosadegh@gmwgroup.harvard.edu

${ }^{\dagger}$ Authors contributed equally

Keywords: Invasion Assay, Migration, Cancer, Oxygen gradients, Chemotaxis 


\section{Abstract}

This work describes a 3D, paper-based assay that can isolate subpopulations of cells based on their invasiveness (i.e., distance migrated in a hydrogel) in a gradient of concentration of oxygen $\left(\mathrm{O}_{2}\right)$. Layers of paper impregnated with a cell-compatible hydrogel are stacked and placed in a plastic holder to form the invasion assay. Stacking the layers of paper assembles them into 3D tissue-like constructs of defined thickness and composition. The plastic holder ensures the layers of paper are in conformal contact; this geometry allows the cells to migrate between adjacent layers through the embedded hydrogel. In most assays, the stack comprises a single layer of paper containing mammalian cells suspended in a hydrogel, sandwiched between multiple layers of paper containing only hydrogel (into which the cells migrate). Cells in the stack consume and produce small molecules; these molecules diffuse throughout the stack to generate gradients both in the stack, and between the stack and the bulk culture medium. Placing the cell-containing layer in different positions of the stack, or modifying the permeability of the holder to oxygen or proteins, alters the profile of the gradients within the stack. Physically separating the layers after culture isolates subpopulations of cells that migrated different distances, and enables their subsequent analysis or culture. Using this system, three independent cell lines derived from A549 cancer cells are shown to produce distinguishable migration behavior in a gradient of oxygen. This result is the first experimental demonstration that oxygen acts as a chemoattractant for cancer cells. 


\section{Significance Statement}

The invasion of cancerous cells from a tumor into surrounding tissues is one contribution to metastasis - a major contributor to death for patients with cancer. There is a strong link between the directed invasion of cancer cells and the gradients of molecules formed in the microenvironment of the tumor. Using a paper-based invasion assay, this work demonstrates that oxygen - a nutrient known to induce significant behavioral changes to cells within a tumor in a concentration-dependent manner - can also act as a chemoattractant, resulting in the migration of cancer cells towards higher concentrations of oxygen. This finding, and the invasion assay described, could lead to a better understanding of oxygen-based chemotaxis in cancer, and ultimately new strategies for managing metastasis. 


\section{Introduction}

Hypoxia - the reduction of levels of oxygen tension to values below normal in a tissue - is toxic to cells $(1,2)$. Cancer cells exposed to hypoxia, both in vitro and in vivo, undergo genetic and/or phenotypic changes that influence cellular metabolism, proliferation, and development of radio/chemo-resistance (3-6). These changes allow the cells to survive in stressful environments. Gradients in concentration of oxygen (we use "oxygen" to mean $\mathrm{O}_{2}$ ) as well as other small molecules (e.g., glucose, nutrients, signaling factors, cellular waste products) $(1-4,6)$ develop normally in tissue due to competition between the supply of oxygen (a process dictated by mass transport) and its consumption by cellular metabolism. These gradients span $\sim 180 \mu \mathrm{m}$ radially from a typical blood vessel. Gradients also develop in solid tumors, but are steeper than those formed in normal tissue due to poorly developed and distributed blood vessels.

Mathematical models have predicted that gradients of oxygen within a tumor direct the migration of cancer cells from the primary tumor to surrounding tissue (5), but to date there has been limited experimental evidence against which to compare these models. This lack of experimental data is due largely to the difficulty in controlling and measuring gradients of oxygen in living tissue, and to the difficulty associated with analyzing the migratory response of cells in conventional model systems (both in vivo and in vitro) (7-11). These mathematical models predict that asymmetric gradients of oxygen and other small molecules such as glucose can cause differential rates of proliferation of cells in a tumor, and these gradients ultimately contribute to the formation of primary tumors with irregular shapes (5). The models also predict an asymmetric migration of cells by the budding and the splitting-off of small populations of cells from the primary tumor. The migration of small groups of cells from the primary tumor is termed collective invasion, and has been observed both in vivo and in vitro (12-18). 
There are a number of methods to assess cellular migration and invasiveness in vitro (9-11, 19-23); these methods rely on quantifying movement across two-dimensional (2D) substrates or through three-dimensional (3D) gels. The concentration gradient of small molecules can be controlled temporally and spatially (at length scales of tens of microns, which is the scale of a single cell) in a microfluidic device $(7,8,24-28)$. These devices are often fabricated in optically transparent materials and make it possible to visualize cell migration in real time (19). Despite the experimental control offered by a microfluidic device, commonly used invasion assayssuch as the Transwell assay (otherwise known as a Boyden chamber), or a Dunn chambersacrifice these precisely defined gradients for ease-of-use, simple end-point readouts, and scalability (9). It is not possible to form gradients of oxygen in a Transwell assay or in a Dunn chamber, and the migratory behavior of cells in response to hypoxic gradients cannot be studied. While a variety of microfluidic devices have been developed to control precise gradients of concentration of oxygen $(7,8,24-29)$, only recently has a device been used to investigate the migration of cells in such gradients (29). Tung et al. demonstrated that oxygen acts as a chemorepellant for A549 cells and these cells migrate toward lower concentrations of oxygen in the presence and absence of an overlapping concentration gradient of stromal cell-derived factor 1 (SDF-1); the gradient of SDF-1 was perpendicular to the concentration gradient of oxygen (29).

To determine if gradients of oxygen direct cellular invasion, we developed an in vitro assay in which cells are cultured in a 3D construct that enables us to analyze cellular migration within a tissue-like environment (Fig. 1A). Unlike most commonly used invasion assays (9, 10, 19, 22, $23,29)$, which impose a gradient of molecules on cells to direct their movement, this assay mimics the mechanism by which gradients are generated in vivo (30-33), and allows the cells to 
consume and secrete molecules within a diffusion-dominated environment.

A paper-based invasion assay_-based on the previously published "Cells-in-Gels-in-Paper", or CiGiP (30-33) — combines the simplicity of commonly used invasion assays with the ability of a microfluidic device to generate gradients of small molecules (albeit with significantly less precision than those generated in a microfluidic device). A unique feature of this assay is the ability to isolate easily cells that migrated different distances by physically separating the layers of the 3D construct (by peeling them apart) after a given period of incubation (Fig. 1). In CiGiP, individual layers of paper are impregnated with cells suspended in a hydrogel and assembled into tissue-like structures by stacking the individual layers. The stack is placed in a holder that is impermeable to gases and proteins. We designed the holder to produce a monotonically decreasing gradient of oxygen from the top of the stack to the bottom. The top of the stack is in contact with bulk culture medium; the bottom of the stack only receives oxygen and nutrients that diffuse (past the cells) from the top of the stack (30-33). This structure, which we refer to in this paper as an "invasion stack" is suitable for assaying cellular invasion for four reasons: i) hydrogels easily wick into and fill the thickness of the paper; ii) the individual layers are in conformal contact, and cells are able to migrate between them; iii) the layers are easily separated from one another, and the location of the cells in the stack (or the distance those cells migrated in the $3 \mathrm{D}$ hydrogel) be assessed without the use of a confocal microscope; and iv) paper is commercially available in a $40 \mu \mathrm{m}$ thickness (Whatman 105), providing a high resolution for distinguishing variable distances migrated by the cells from their original position $(32,33)$.

This manuscript demonstrates that invasion assays can be performed in the $3 \mathrm{D}$ culture environment of the CiGiP system. Using the invasion stacks, we demonstrate that subpopulations of the A549 cancer cell line undergo directed movement toward higher 
concentrations of oxygen (i.e., chemotaxis). This result is, to our knowledge, the first demonstration that different cancer cell lines are distinguishable by their migratory response to a gradient of concentrations of oxygen. While the exact mechanism of cellular movement (and thus invasion) in these gradients of concentrations of oxygen is not understood, it may lead to a better understanding of strategies used in cancer therapies, and provide a model system to elucidate the migratory behaviors of mammalian cells in $3 \mathrm{D}$ environments.

\section{Results}

Cancer cells readily invade multiple layers of the invasion stack. In a standard experiment, we assembled nine layers of wax-patterned paper into an invasion stack (Fig. 1A). A single layer of paper containing a suspension of cells in a hydrogel (a "seeded layer", labeled "layer 0") was sandwiched between layers of paper containing only hydrogel. We refer to the layers containing only hydrogel as the "invaded layers" and label each with a "+" or "_" and a number to signify its position relative to the seeded layer (the "+" indicates a layer closer to the top of the stack and the oxygen-containing medium). The details of fabricating wax-printed layers of paper and culturing mammalian cells within stacks are similar to assays based on CiGiP, which have been described previously (30-33); a detailed description of the procedures used in this work can be found in the Supplemental Information.

Each layer contained 20 hydrophilic zones surrounded by hydrophobic wax boundaries. The ability to examine multiple zones in a single assay allowed us to carry out replicate experiments in parallel, and obtain statistically relevant data from a single invasion stack. Ten of the zones in each layer contained neither hydrogel nor cells suspended in hydrogel; these "blank" zones allowed us to determine the fluorescence background of each layer of paper (Fig. 1A), and 
aided in data analysis.

We cultured the invasion stacks in poly(methyl methacrylate) (i.e., "acrylic") holders, which ensured the layers were in conformal contact throughout the experiment (Fig. 1B). This configuration allowed cells to invade vertically between adjacent layers, but not laterally between zones of a given layer. Additionally, the impermeability of the acrylic holders to oxygen and other molecules allowed us to control the mass transport of oxygen and nutrients from the bulk culture medium to the layers of the invasion stack (30-33).

In this paper, we compare three types of A549 cells in the invasion stacks: i) A549, which are basal epithelial cells of a human alveolar adenocarcinoma (34); ii) A549-HGF, which are A549 cells engineered to express hepatocyte growth factor (HGF) constitutively $(35,36)$; and iii) A549-HGF-M, which is a subclone of the A549-HGF cells derived from a lung metastasis in a xenograft tumor grown in an immune-compromised mouse.

After culturing the stack in serum-containing medium for a given period of time, we disassembled the holder, separated the nine layers of the invasion stack, and imaged each layer in parallel with a commercially available flatbed fluorescence scanner. Figure 1C contains images of a representative invasion stack of GFP-expressing A549-HGF-M cells after 24 hours of culture (initially only layer 0 contained cells). Cell-containing zones are in a checkerboard pattern with only two zones of the first row of the image in Figure 1D containing cells. Darker regions correspond to increased fluorescence intensity and a larger number of GFP-expressing cells; darker regions in the zone correlate with a higher density of cells. The pattern of the hydrophobic wax is also visible in the image, and is due to the autofluorescence of the wax.

Cells invade not only layers in direct contact with the seeded layer (i.e., layer +1 and layer -1 ), but also layers that are further than two positions from the seeded layer; distances of $>80$ 
microns. Cells in these distant layers are in aggregates despite having significant space within the zone, while the more populated layers (i.e., layers $-1,0,+1$ ) fill the zone uniformly (Fig. 1C). This observation suggests these subpopulations of cells invade in a multi-cellular manner, indicative of collective invasion and multi-cellular streaming (see Supplemental Figure 1 for high-resolution images) (15).

Cellular invasion can be imaged and quantified in the invasion stack. To quantify the distribution of cells in the invasion stack, we collected a fluorescent image of each layer of paper in the stack after 24 hours of culture (Fig. 1C). We measured the fluorescence intensity of each zone with ImageJ, and calculated the "background fluorescence intensity" for each layer as the average fluorescence intensity of the 10 blank zones plus three times their standard deviation. If the fluorescence intensity of a cell-containing zone was less than the background fluorescence intensity for a given layer, then we set its fluorescence value to zero. Figure 1E shows a plot of the distribution of fluorescence intensities of each cell-containing zone for the images shown in Figure 1C. The distribution is normalized to the total fluorescence intensity of the stack, and displays the average fluorescence value for all of the zones as a green bar and the variation of fluorescence between the zones as a black error bar, which corresponds to one standard deviation.

In addition to the low-resolution fluorescent images, we also obtained confocal images of the cells in the different layers of the invasion stack. Figure $1 \mathrm{~F}$ is a representative confocal image of the cells from one of the zones in layer 0 of Figure 1C. The image shows the cells are suspended in hydrogel with minimal interaction between the cells and the fibers of the paper. The fibers of the paper appear blue due to their autofluorescence upon excitation with UV light. 


\section{Metastatic subclone of A549 cells shows more chemotactic behavior than parental cell}

lines in the paper-based invasion stacks. To determine if cells of different metastatic potential displayed similar trends in invasiveness in vitro and in vivo, we compared the invasiveness of the A549, A549-HGF, and A549-HGF-M cells in the invasion stack and in immune-compromised mice.

We assembled invasion stacks in the configuration shown in Figure 1; the seeded layer contained 100,000 cells/zone. Each invasion stack was incubated for 24 hours in an acrylic holder, which only permitted the exchange of nutrients between the bulk culture medium and the top of the stack. The distribution of each type of cell compiled from three separate invasion stacks is shown in Figure 2 (each stack contained 10 replicate zones, and the mean values and standard deviations are for all 30 replicates). For each type of cell, a large percentage of cells remained in the seeded layer (> 20\%). The distribution of the A549 and A549-HGF cells was symmetric around the seeded layer with no statistical difference in the percentage of cells in layers +1 and -1 . These data suggest the cells invaded the neighboring layers as a result of either chemokinesis or from a crowding effect of cells in the seeded layer (37).

The distribution of A549-HGF-M cells in the invasion stack was asymmetric, with: $50 \% \pm$ $2 \%$ of all cells in the invasion stack migrating upwards toward the source of nutrients, $28 \% \pm 1 \%$ of all cells in the invasion stack migrating downwards away from the source of nutrients, and 22 $\pm 3 \%$ of all cells remaining in the seeded layer. Each of the types of cells lines also contained a detectable subpopulation of cells ( $1 \%$ of the total number of cells in the invasion assay) in the layer closest to the source of fresh medium (layer +4$)$.

To determine whether the observed differences between these three types of cells in our invasion assay correlate to the potential of these cells to form metastatic colonies in the lung of 
xenograft models, we implanted equal numbers of each cell line subcutaneously into immunecompromised mice. We measured the volume of each tumor and counted the number of metastatic clones (mets) in the lungs of each mouse 22 days after implantation ( $\mathrm{n}=10$ mice), or once the volume of the primary tumor reached $1,000 \mathrm{~mm}^{3}(\mathrm{n}=10-12 \mathrm{mice})$. A detailed experimental protocol for the implantation and analysis of the metastatic clones, as well as representative images obtained from the mice, are in the SI.

Over a period of 22 days, the parental A549 tumors (with volumes of $404 \pm 139 \mathrm{~mm}^{3}$ ) generated significantly fewer mets $(\sim 1 \pm 1$ mets $)$ than the other two cell line-derived xenograft tumors $(\mathrm{P}<0.0001)$; this observation suggests the parental A549 cells have the lowest metastatic potential in vivo (Fig. S2E). There was no statistical difference between the number of lung mets measured in mice bearing tumors derived from A549-HGF (73 \pm 79 mets, $\left.1512 \pm 594 \mathrm{~mm}^{3}\right)$ and A549-HGF-M cells (39 \pm 40 mets, $1905 \pm 1031 \mathrm{~mm}^{3}$ ). The parental A549 cells, however, generated statistically indistinguishable numbers of mets ( $46 \pm 36$ mets) when the tumors were allowed to grow to similar sizes as the tumors generated from A549-HGF and A549-HGF-M cells (Fig. S2F). All three types of cells, therefore, have equal metastatic potential in the long term, which is a result that correlates with the presence of rapidly moving cells (cells in layer +4$)$ in all three types of A549 cells in the invasion assay.

The presence of more cells in layer +1 than layer -1 in the invasion stack for the A549HGF-M cells (Fig. 2C), does not seem to correlate to the presence of additional mets in the lungs of the mice. We, however, do not yet know how this subpopulation of slowly moving cells that undergo chemotaxis towards higher concentrations of oxygen may increase the metastatic potential of the population in: i) immunocompetent mice, ii) humans, iii) organs other than the lung, iv) implantations of longer duration than weeks, and v) implantations to sites other than 
subcutaneous.

The subpopulation of cells that migrated preferentially toward bulk culture medium in the invasion assay raises four questions: i) Is the distribution of the cells in the invasion stack a result of chemokinesis (random migration within a chemical stimuli), chemotaxis (migration of cells in response to a gradient of concentration of a chemical), or a combination of the two? ii) Is the distribution an artifact of cells proliferating at different rates between layers experiencing different oxygen tensions due to their location in the stack? iii) If the cells in the invasion stack are undergoing chemotaxis, which component(s) in the medium (i.e., oxygen, glucose, autocrine factors, waste products) is (are) the main driver(s) of this preferential migration? iv) Are there differences in a given population of cells that pre-dispose some cells to be highly invasive and others to be less invasive, and can we use the invasion stack to separate these different subpopulations?

We carried out experiments with the invasion stacks to address each of these questions, and discuss our results in the following sections.

Highly invasive cells are distinguishable after a short period of culture. To estimate the rate at which the highly invasive subpopulations of HGF-expressing cells migrate from layer 0 to layer +4 of the invasion assay (Fig. $2 \mathrm{~B}$ and $2 \mathrm{C}$ ), we monitored the migration of the A549-HGFM cells as a function of time. Each stack was cultured under the same conditions - the seeded layers contained 100,000 cells/zone, and the stack was cultured in the acrylic holder shown in Figure 1B—but destacked after an incubation period of 1, 12, or 48 hours.

After 1 hour of culture (Fig. 3A), three separate sub-populations of cells emerge: i) highly invasive cells that quickly migrate from the seeded layer to the layer closest to the source of 
fresh medium ( $4 \% \pm 5 \%$ of total cells in the assay); ii) invasive cells that migrate both toward and away from the source of fresh medium equally ( $20 \% \pm 13 \%$ of total cells in the assay); and iii) non-invasive cells that do not migrate from the seeded layer $(77 \% \pm 23 \%$ of total cells in the assay). Increased periods of culture increased the percentage of invading cells to total cells in the assay from $20 \% \pm 13 \%$ (after 1 hour of culture, Fig. 3A) to $80 \% \pm 6 \%$ (after 12 hours of culture, Fig.3B) and $90 \% \pm 2 \%$ (after 48 hours of culture, Fig. 3C), respectively. The ratio of cells invading toward the source of nutrients versus away from the source of nutrients also increased progressively (from $1.6 \pm 0.2$ at 12 hours, and $3.0 \pm 0.5$ at 48 hours of culture). We note that changes in the proliferation rate of cells in different layers of the invasion stack may affect the final distribution of cells in the stack for prolonged periods of culture (i.e., cells closer to the source of nutrients could be proliferating faster).

These results show that a subpopulation $(\sim 4 \% \pm 5 \%)$ of the A549-HGF-M cells undergo rapid migration in the invasion stack and migrate $160 \mu \mathrm{m}$ in 1 hour. The more slowly moving A549-HGF-M cells, migrate preferentially towards the source of nutrients but still migrate outward in both directions from the seeded layer; this suggests that both chemotaxis and crowding play a role in the final distribution of the cells. To determine the effect of crowding on invasiveness in our system, we investigated the effect of seeding density on the distribution of A549-HGF-M cells in the invasion stack.

\section{Increasing the density of cells in the seeded layer increased short-range invasiveness}

but decreased long-range invasiveness. To determine if the cellular density of the seeded layer influenced the final distribution of invading cells, we incubated invasion stacks containing $10 \mathrm{x}$ $10^{3}, 33 \times 10^{3}$, or $100 \times 10^{3}$ A549-HGF-M cells/zone for 24 hours (Fig. 3 D-F). The percentage of 
invading cells to total cells in the invasion assay increased progressively from $25 \% \pm 7 \%$ for a seeding density of $10 \times 10^{3}$ cells/zone to $45 \% \pm 14 \%$ for $33 \times 10^{3}$ cells/zone, and $80 \% \pm 5 \%$ for $100 \times 10^{3}$ cell/zone.

We observed a population of cells that moved rapidly to layer +4 for all three seeding densities, but the lowest seeding density $\left(33 \times 10^{3}\right.$ cells/zone $)$ had a significantly larger percentage of cells $(4.5 \%$ versus $1 \%)$ in layer +4 than the highest seeding density. We only observed a statistically significant bias $(\mathrm{p}<0.01)$ in the distribution of slowly moving cells (layers +1 and -1 ) for cell seeding densities of $33 \times 10^{3}$ cells/zone (Fig. 3E) and $100 \times 10^{3}$ cells/zone (Fig. 3F). This result suggests that the slowly moving subpopulation of cells undergo chemotaxis only for larger seeding densities. To validate further that chemotaxis was occurring in the invasion stacks, we placed the seeded layer in different positions of the invasion stack and placed the cells at opposing ends of the gradients.

Metastatic cells undergo chemotaxis in the invasion stack. The biased migration of the A549-HGF-M cells toward the source of nutrients suggests that a subpopulation of cells in the invasion stack undergo chemotaxis. To validate this hypothesis, we compared the extent of cellular invasion when the seeded layer was positioned at either the top or bottom of the invasion stack. A comparison of Figures 4A (seeded layer positioned at the top of the stack) and 4B (seeded layer positioned at the bottom of the stack) indicates invasion is chemotactic because a larger percentage of cells moved toward the source of nutrients $(61 \% \pm 6 \%$ of the cells in the seeded layer, Fig. 4B) than away from the source of nutrients $(12 \% \pm 3 \%$ of the cells in the seeded layer, Fig. 4A).

Gradients of molecules that may elicit chemotaxis of cells in the invasion stacks could be 
formed by consumption of molecules present in the medium (e.g., oxygen, glucose) or generation of molecules secreted by the cells (e.g., signaling molecules, $\mathrm{HCO}_{2}{ }^{-}, \mathrm{H}^{+}$, and other waste products). We, therefore, engineered holders that would allow us to determine whether oxygen was the primary cause for chemotaxis in the invasion stack.

Oxygen is the primary chemo-attractant in the invasion stack. To determine if oxygen was the primary chemoattractant inducing the directed movement we observed in the invasion stacks of A549-HGF-M cells, we decoupled the gradient of oxygen from three other possible candidates: proteins from the medium, autocrine factors, and waste products.

We engineered two variants of the holder shown in Figure 1. In the first variant, both the top and bottom plate contained a series of holes that were permeable to both gases and proteins, and permitted exchange between bulk culture medium and the top and bottom of the invasion stack (Fig. 4C). In the second variant, the top and bottom plate also contained a series of holes; the holes in the bottom plate were sealed with a $200 \mu \mathrm{m}$ thick layer of poly(dimethyl)siloxane (PDMS). PDMS is a gas-permeable material and allowed the exchange of oxygen and $\mathrm{CO}_{2}$ between the bulk culture medium and the bottom of the stack, but prevented the diffusion of other small molecules. Since oxygen should have a symmetric gradient of concentrations in both types of invasion stacks, and proteins should have an asymmetric gradient of concentrations in the second invasion stack but not the first, we could determine if oxygen was the primary chemoattractant if the cells showed a symmetric distribution in both stacks. Figure 4C and 4D do, indeed, show symmetric distributions. These results, overall, suggest that the gradient of oxygen formed in the invasion stack is the primary chemoattractant for this system (i.e., number of layers, thickness of paper, density of cells, and type of cell). 
The percentage of highly invasive cells is dictated by the culture environment, and is not a result of genetic or phenotypic predisposition of a subpopulation of cells. To

determine if the sub-population of the highly invasive HGF-expressing cells, which migrated to layer +4 had a stable genotype or phenotype that was intrinsically more invasive than the cells that remained in the seeded layer, we recovered A549-HGF cells isolated from layer 0 and layer +4 from an invasion stack and compared their invasiveness in subsequent invasion stacks. We expanded the two subpopulations of cells in separate 2D culture flasks (the number of isolated cells was too few to perform an invasion assay) and then seeded them into two separate layers at a density of 100,000 cells/zone (Fig. 5A). We assembled invasion stacks for each type of cell in the format shown in Fig. 1B and cultured the stacks for 24 hours.

The distribution of cells in the invasion stacks of these two different populations of cells was nearly indistinguishable (Fig. 5B). These results suggest that the rapidly invading cells do not maintain a stable phenotype in 2D culture. Other cancer cell lines or primary cells may behave differently and therefore this assay could be used to isolate, enrich, and characterize cells of a specific phenotype (i.e., cancer stem cells).

\section{Discussion.}

This work describes results obtained from a newly developed paper-based invasion assay in which: i) We demonstrate the ability to isolate and recover sub-populations of cells based on the distance migrated in the assay. ii) Cell lines with a higher metastatic potential in vivo undergo chemotaxis rather than chemokinesis in response to the gradient of oxygen formed during culture. iii) The highly migratory phenotype of subpopulations of A549 cells within the 
metastatic cell lines is not stable under 2D culture, and this phenotype may be dependent on the specific niche conditions within the invasion stack.

This paper-based assay has several attractive characteristics $(9-11,19-23)$ that make it useful in studying cellular invasion. i) Easy to assemble. We pipette suspensions of cells in the liquid phase of a hydrogel, and allow the gel to form around the cellulose fibers of the paper so that 40micron thin layers of hydrogel can be manipulated manually. Multiple layers are assembled to form invasion stacks of defined thickness. These manipulations are straightforward to perform. ii) Modular in design. We controlled the distribution of cells seeded in the invasion stack simply by changing the components (e.g., types of cells, density of cells) in each layer. iii) Easy to analyze, and to recover, cells from the layers. The ability to separate the layers of paper at the end of an experiment allows us to analyze and compare the population of cells in each layer. The cells remain viable after separation, and would be recovered for further analysis or continued culture. We are able to image the cells in each sheet of paper with a fluorescence scanner (which provides high-throughput, but low-resolution data; Fig. 1C) or a confocal microscope (which provides low-throughput, but high-resolution data; Fig. 1F).

Current in vitro studies of cellular response to changes in concentrations of oxygen rely on hypoxia chambers, which regulate the bulk concentration of oxygen to which a culture of cells is exposed. Furthermore, hypoxia chambers do not generating gradients of oxygen to study chemotaxis. A well-defined gradient of oxygen can be formed in a microfluidic device containing cells, but these devices are predominantly used to study cellular viability, proliferation, and differentiation $(7,8,24-28)$. To our knowledge, in vitro studies of chemotaxis in the presence of a gradient of oxygen — particularly one generated by cellular metabolismhave yet to be performed for mammalian cells. The fact that such studies have not been 
performed is due not to a disinterest in the role of oxygen in tumor migration, but to the limited utility of most microfluidic devices in the hands of cell biologists.

We speculate that a paper-based assay may be more useful for cell culture than microfluidic devices for these three reasons: i) An invasion stack is easier to set up than most microfluidic assays. ii) A higher volume of statistically relevant data can be more easily generated in the invasion stacks than in most microfluidic devices. iii) Sub-populations of cells can be easily recovered from the layers of paper for further analysis (e.g, FACS, qPCR), while the recovery of subpopulations of cells from a microfluidic device can be difficult.

The current, paper-based assay has two primary drawbacks: i) The migration of the cells cannot be tracked in real time, and thus tracking the migration of single cells is difficult. ii) The gradients formed in the stack are currently difficult to measure directly. These limitations, however, can be overcome with further development, characterization, and engineering of the assay.

Our findings that the migratory response of a population of cells within a gradient of oxygen correlates with its metastatic potential in vivo may have value for angiogenic-based therapies (38-40). This correlation is in agreement with current literature and mathematical models, and suggests the invasion of cancer cells is driven largely by the metabolic needs of a tumor (4-6, 41). Furthermore, the versatility of this assay has the potential for investigating other important factors in metastasis, such as effects of gradients of cytokines, co-cultures of multiple types of cells, and invasion through extracellular matrices of varying composition. We believe this versatility coupled with the ability to separate sub-populations of cells from the paper-based invasion assay may enable discovery of novel biomarkers and better testing of 3D invasion assays for drug screening (42). 
Oxygen as a chemokine for mammalian cells is a largely unstudied field of biology. To our knowledge, only one other work experimentally evaluates the migration of mammalian cells in gradients of oxygen (29). Tung et al. used a microfluidic device that exposed A549 cells to gradients of oxygen ranging from $3.8 \%$ to $13.6 \%$. A549 cells in this device showed a chemotactic response that moved toward lower concentrations of oxygen. This result is contradictory to the behavior of A549 cells in our invasion stacks, which moved toward atmospheric levels of oxygen (i.e., 20\%). There are significant differences between these two migration assays that could account for the different migratory behavior of the A549 cells: i) 2D migration in the microfluidic device versus 3D invasion in the invasion stack, ii) autocrine factors are continuously removed from the microfluidic device, but maintained within the invasion stack, iii) the microfluidic device imposes a gradient of oxygen on the cells, but the invasion stack allows the metabolism of the cells to generate the gradient of oxygen as in done in vivo, iv) the density of cells in the microfluidic device (2000 cells/ $\mu \mathrm{l})$ is lower than the invasion stack $(100,000$ cells $/ \mu \mathrm{l})$. The experimental differences in these systems are known to have significant effects on migratory behavior of cells and alter many downstream gene expression profiles $(10,43,44)$. Since the mechanism of how cells sense gradients of oxygen, and use that information to migrate toward a particular direction, is not well understood, the differences listed between these two systems may or may not account for the difference in behavior. We believe, however, that the discrepancy between these two studies should stimulate research intended to understand the mechanisms involved with chemotaxis of cells in gradients of oxygen and how they regulate behavior in vivo in both physiological and pathological environments. 


\section{Materials and Methods}

The materials and methods used in this work are summarized here, and presented in detail in the Materials and Methods section of the Supporting Information. Cell Culture. All lines of A549 cells were cultured at $37{ }^{\circ} \mathrm{C}$ and $5 \% \mathrm{CO}_{2}$ in DMEM medium containing $10 \%$ fetal bovine serum and 1\% PenStrep. Fabrication of Paper Layers. Layers of paper were fabricated using protocols detailed in (32). Statistical Analysis. Data are presented as mean \pm SD. Student's $t$ test (two-tailed) was used to compare two groups ( $\mathrm{P}<0.05$ was considered significant) unless otherwise indicated.

\section{Acknowledgements}

This work was supported through a collaborative research agreement between Vertex Pharmaceuticals, Inc. (Boston, MA) and Harvard University. The Wyss Institute for Biologically Inspired Engineering partially funded the salary for BM, and provided microscopy tools. KG thanks the Biomedical Research Initiative Dedicated to Gateway Experiences (NSF DMR-1005022). We acknowledge Ratmir Derda and Renee Fuller for performing initial experiments involving a paper-based invasion assay. 


\section{References}

1. Wilson WR \& Hay MP (2011) Targeting hypoxia in cancer therapy. Nat Rev Cancer 11(6):393-410.

2. Harris AL (2002) Hypoxia - A key regulatory factor in tumour growth. Nat Rev Cancer 2(1):38-47.

3. Erickson K, et al. (2003) Effect of longitudinal oxygen gradients on effectiveness of manipulation of tumor oxygenation. Cancer Res 63(15):4705-4712.

4. Han TY, et al. (2013) How does cancer cell metabolism affect tumor migration and invasion? Cell Adh Migr 7(5):395-403.

5. Cristini V, et al. (2005) Morphologic instability and cancer invasion. Clin Cancer Res 11(19):6772-6779.

6. Carmona-Fontaine C, et al. (2013) Emergence of spatial structure in the tumor microenvironment due to the Warburg effect. Proc Natl Acad Sci USA 110(48):19402-19407.

7. Oppegard SC \& Eddington DT (2013) A microfabricated platform for establishing oxygen gradients in 3-D constructs. Biomed Microdevices 15(3):407-414.

8. Lo JF, Sinkala E, \& Eddington DT (2010) Oxygen gradients for open well cellular cultures via microfluidic substrates. Lab Chip 10(18):2394-2401.

9. Sackmann EK, Fulton AL, \& Beebe DJ (2014) The present and future role of microfluidics in biomedical research. Nature 507(7491):181-189.

10. Mosadegh B, Saadi W, Wang SJ, \& Jeon NL (2008) Epidermal growth factor promotes breast cancer cell chemotaxis in CXCL12 gradients. Biotechnol Bioeng 100(6):12051213.

11. Torisawa YS, et al. (2010) Microfluidic platform for chemotaxis in gradients formed by CXCL12 source-sink cells. Integr Biol 2(11-12):680-686.

12. Cheung Kevin J, Gabrielson E, Werb Z, \& Ewald Andrew J (2013) Collective invasion in breast cancer requires a conserved basal epithelial program. Cell 155(7):16391651.

13. Khalil AA \& Friedl P (2010) Determinants of leader cells in collective cell migration. Integr Biol 2(11-12):568-574.

14. Graham JM \& Ayati BP (2012) A unified term for directed and undirected motility in collective cell invasion. Appl Math Lett 25(12):2267-2271.

15. Friedl P, Locker J, Sahai E, \& Segall JE (2012) Classifying collective cancer cell invasion. Nat Cell Biol 14(8):777-783.

16. Wan LL, Pantel K, \& Kang YB (2013) Tumor metastasis: moving new biological insights into the clinic. Nat Med 19(11):1450-1464.

17. Dona E, et al. (2013) Directional tissue migration through a self-generated chemokine gradient. Nature 503(7475):285-289.

18. Tarabichi M, et al. (2013) Systems biology of cancer: entropy, disorder, and selection-driven evolution to independence, invasion and "swarm intelligence". Cancer Metastasis Rev. 32(3-4):403-421.

19. Keenan TM \& Folch A (2008) Biomolecular gradients in cell culture systems. Lab Chip 8(1):34-57. 
20. Mahmud G, et al. (2009) Directing cell motions on micropatterned ratchets. Nat Phys 5(8):606-612.

21. Jiang XY, Bruzewicz DA, Wong AP, Piel M, \& Whitesides GM (2005) Directing cell migration with asymmetric micropatterns. Proc Natl Acad Sci USA 102(4):975-978.

22. Boyden S (1962) Chemotactic effect of mixtures of antibody and antigen on polymorphonuclear leucocytes. J Exp Med 115(3):453-466.

23. Zicha D, Dunn GA, \& Brown AF (1991) A new direct-viewing chemotaxis chamber. J Cell Sci 99(4):769-775.

24. Oppegard SC, Blake AJ, Williams JC, \& Eddington DT (2010) Precise control over the oxygen conditions within the Boyden chamber using a microfabricated insert. $L a b$ Chip 10(18):2366-2373.

25. Polinkovsky M, Gutierrez E, Levchenko A, \& Groisman A (2009) Fine temporal control of the medium gas content and acidity and on-chip generation of series of oxygen concentrations for cell cultures. Lab Chip 9(8):1073-1084.

26. Allen JW \& Bhatia SN (2003) Formation of steady-state oxygen gradients in vitro Application to liver zonation. Biotech Bioeng 82(3):253-262.

27. Adler M, Polinkovsky M, Gutierrez E, \& Groisman A (2010) Generation of oxygen gradients with arbitrary shapes in a microfluidic device. Lab Chip 10(3):388-391.

28. Chen YA, et al. (2011) Generation of oxygen gradients in microfluidic devices for cell culture using spatially confined chemical reactions. Lab Chip 11(21):3626-3633.

29. Chang C-W, et al. (2014) A polydimethylsiloxane-polycarbonate hybrid microfluidic device capable of generating perpendicular chemical and oxygen gradients for cell culture studies. Lab Chip 14(19):3762-3772.

30. Deiss F, et al. (2013) Platform for high-throughput testing of the effect of soluble compounds on 3D cell cultures. Anal Chem 85(17):8085-8094.

31. Derda R, et al. (2009) Paper-supported 3D cell culture for tissue-based bioassays. Proc Natl Acad Sci USA 106(44):18457-18462.

32. Derda R, et al. (2011) Multizone paper platform for 3D cell cultures. PLoS One 6(5):e18940.

33. Mosadegh B, et al. (2014) Three-dimensional paper-based model for cardiac ischemia. Adv Healthc Mater 3(7):1036-1043.

34. Giard DJ, et al. (1973) In vitro cultivation of human tumors: establishment of cell lines derived from a series of solid tumors. J Natl Cancer Inst 51(5):1417-1423.

35. Ramanujum R, Lin Y-L, Liu J-K, \& He S (2013) Regulatory expression of MMP8/MMP-9 and inhibition of proliferation, migration and invasion in human lung cancer A549 cells in the presence of HGF variants. Kaohsiung J Medical Sci 29(10):530-539.

36. Gumustekin M, et al. (2012) HGF/c-Met Overexpressions, but not Met Mutation, Correlates with Progression of Non-small Cell Lung Cancer. Pathol Oncol Res 18(2):209-218.

37. MC L, SR V, CT L, \& B L (2013) Geometrical constraints and physical crowding direct collective migration of fibroblasts. Commun Integr Biol 6(2):e23197.

38. Potente M, Gerhardt H, \& Carmeliet P (2011) Basic and Therapeutic Aspects of Angiogenesis. Cell 146(6):873-887.

39. Scianna M, Bell CG, \& Preziosi L (2013) A review of mathematical models for the formation of vascular networks. J Theor Biol 333:174-209. 
40. Goel S, et al. (2011) Normalization of the vasculature for treatment of cancer and other diseases. Physiol Rev 91(3):1071-1121.

41. Chen J, Sprouffske K, Huang QH, \& Maley CC (2011) Solving the Puzzle of Metastasis: The Evolution of Cell Migration in Neoplasms. PloS One 6(4):e17933.

42. Weigelt B, Ghajar CM, \& Bissell MJ (2014) The need for complex 3D culture models to unravel novel pathways and identify accurate biomarkers in breast cancer. $A d v$ Drug Deliv Rev 69-70:42-51.

43. Kay RR, Langridge P, Traynor D, \& Hoeller $O$ (2008) Changing directions in the study of chemotaxis. Nat Rev Mol Cell Biol 9(6):455-463.

44. Baumann K (2010) Cell migration: Moving in 3D. Nat Rev Mol Cell Biol 11(7):465465. 

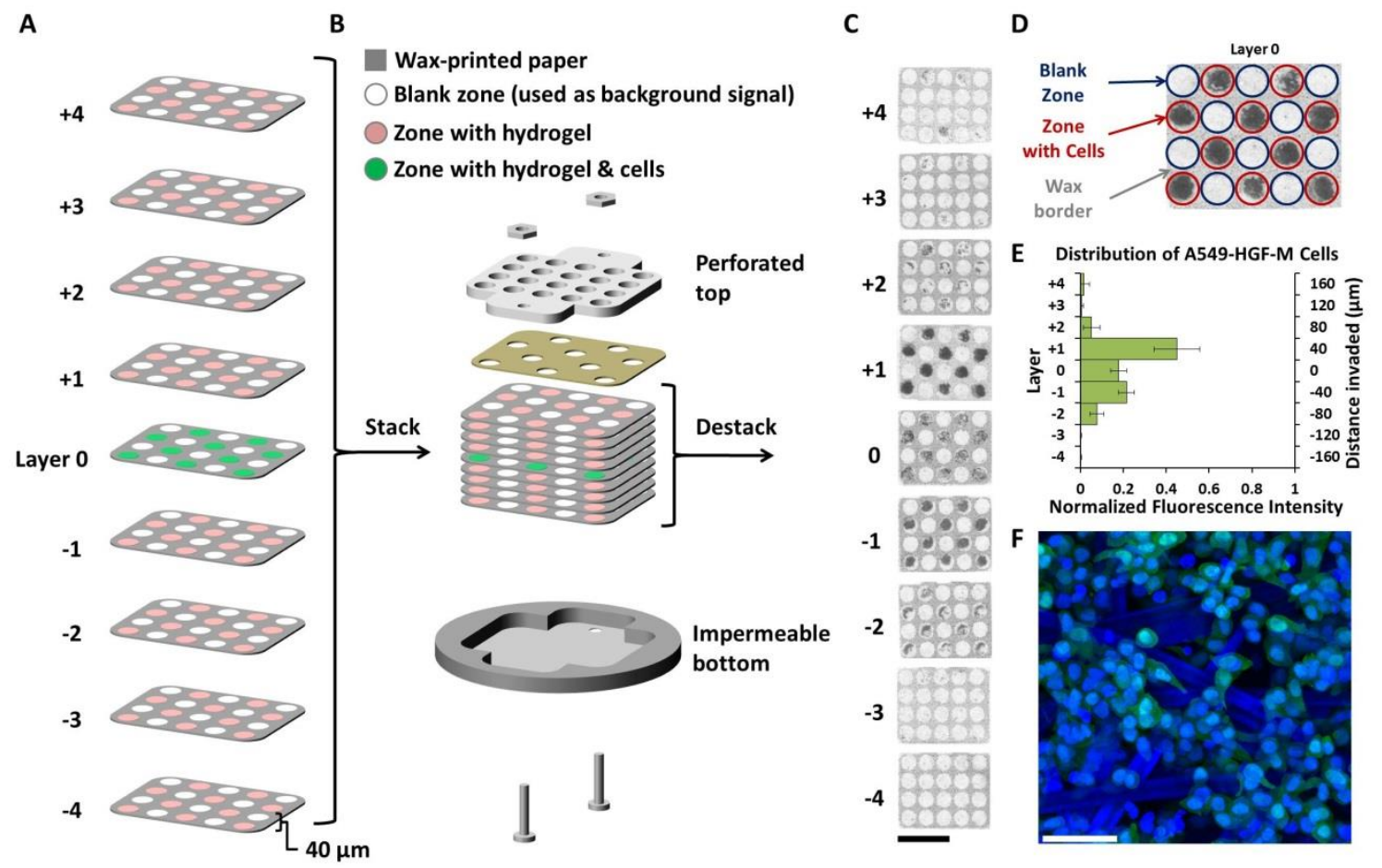

Figure 1. Paper-based invasion assay. A) Schematic of a single layer of wax-patterned paper (40 $\mu \mathrm{m}$ thick) containing GFP-expressing A549-HGF-M cells suspended in a hydrogel (Layer 0) positioned between sheets of paper containing only hydrogel (Layers $0+n$ for layers above the seeded layer, and $0-n$ for layers below the seeded layer). B) Schematic of all layers of paper stacked with a laser-cut transparency in an acrylic holder using two screws and nuts. C) Images from a fluorescent scanner of all layers of paper after 24 hours in culture. An array of circular zones is visible due to the autofluorescence of the wax. Within the circles, darker pixels correlate with a higher intensity of GFP (e.g., higher density of cells). Scale bar: $1 \mathrm{~cm}$. D) Image of layer 0 with red circles outlining cell-seeded zones and blue circles outlining blank zones, which are used to determine background intensity of the layer. E) Plot of the average intensity of GFP within each layer, normalized by the total intensity of GFP within all layers of the stack. Each layer was cultured separately overnight and then stacked for 24 hours before being destacked and imaged. Error bars represent the standard deviation for 10 replicate zones within the same layer. F) Confocal image of cells within layer 0: green is GFP (A549-HGF-M), blue is DAPI (nuclei). Fibers of the paper are visible due to their autofluorescence under ultraviolet irradiation. Scale bar: $50 \mu \mathrm{m}$ 

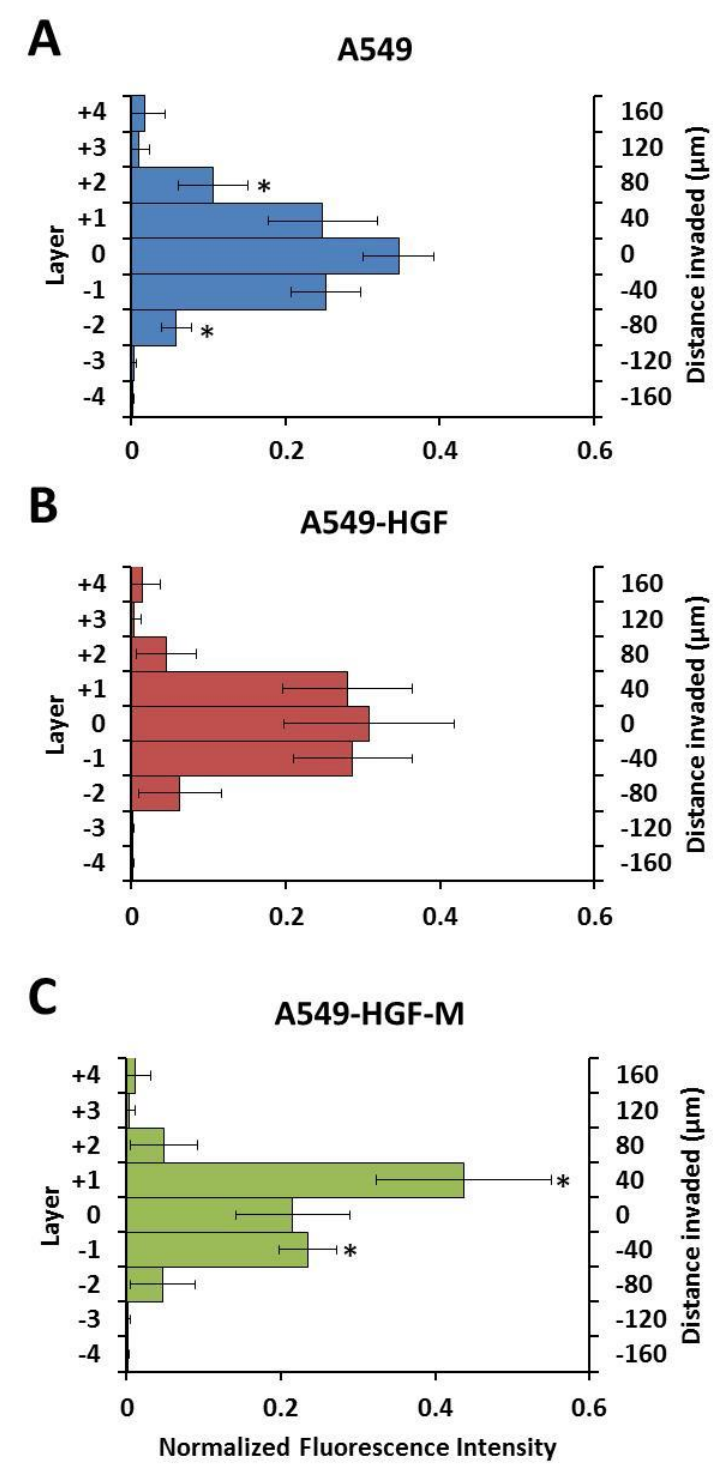

Figure 2. Invasiveness of different types of A549 cells in the paper-based invasion stack. A-C) Cellular distribution of A549 (A), A549-HGF (B), and A549-HGF-M (C). Cells were initially seeded in layer 0 at 100,000 cells/zone, incubated overnight, stacked, and then cultured for 24 hours before imaging. Error bars represent the standard deviation for three separate invasion assays with a total of 30 replicate zones. ${ }^{*}$ represents a $\mathrm{p}<0.01$. 


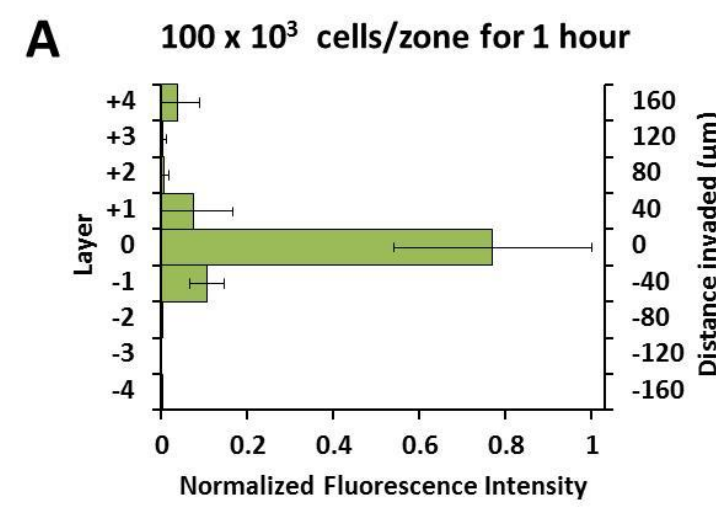

B $100 \times 10^{3}$ cells/zone for 12 hours

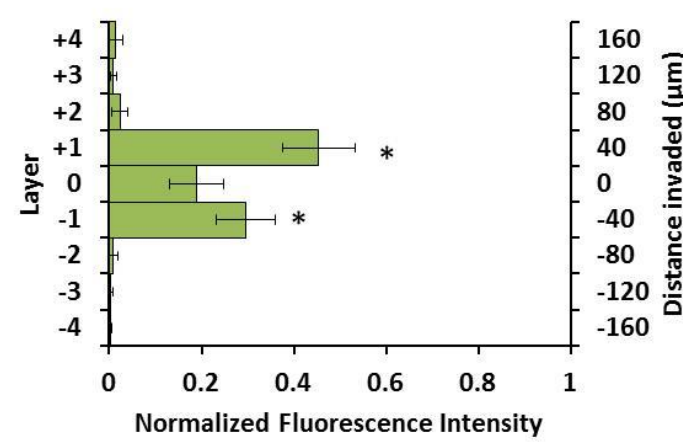

C $100 \times 10^{3}$ cells/zone for 48 hours

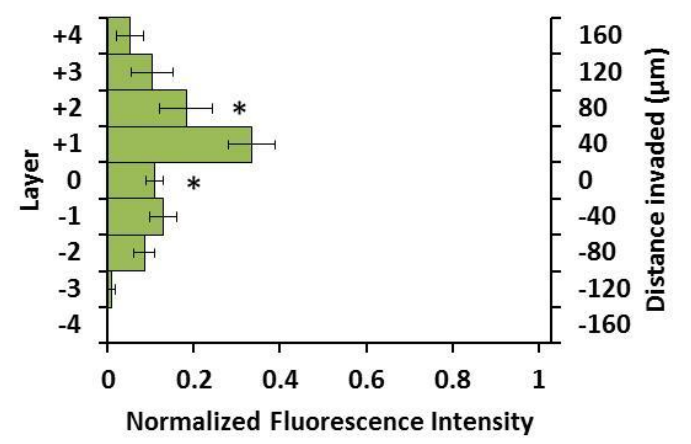

D $\quad 10 \times 10^{3}$ cells/zone for 24 hours

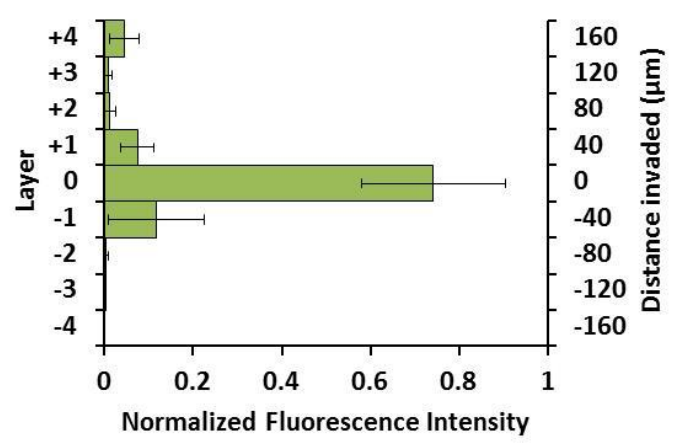

E $33 \times 10^{3}$ cells/zone for 24 hours

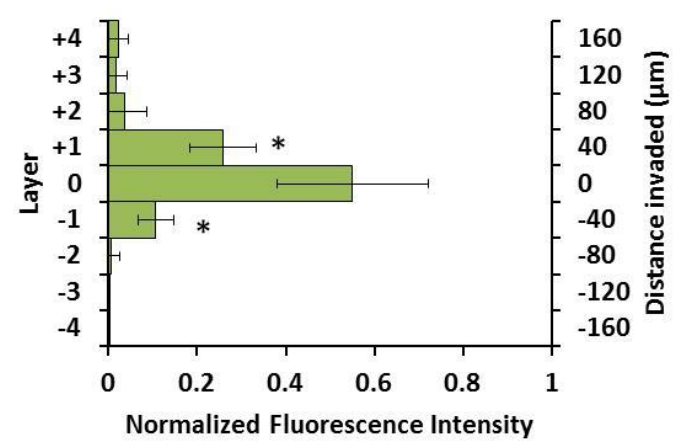

F $\quad 100 \times 10^{3}$ cells/zone for 24 hours

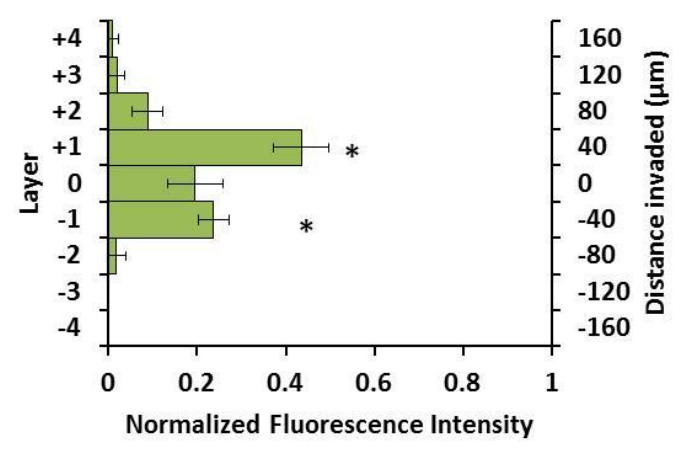

Figure 3. Rapidly and slowly moving populations of A549-HGF-M cells are resolved with shorter durations of culture. A-C) Distribution of A549-HGF-M cells within the invasion assay with increasing periods of culture. A549-HGF-M cells were cultured overnight prior to stacking (100,000 cells/zone). D-F) Distribution of A549-HGF-M cells in the invasion assay after 24 hours for a seeded density of $10 \times 10^{3}, 33 \times 10^{3}$, or $100 \times 10^{3}$ cells/zone. Cells were cultured overnight prior to stacking. Error bars represent the standard deviation for either 10 or 20 replicate zones. ${ }^{*}$ represents a $\mathrm{p}<0.01$. 
A

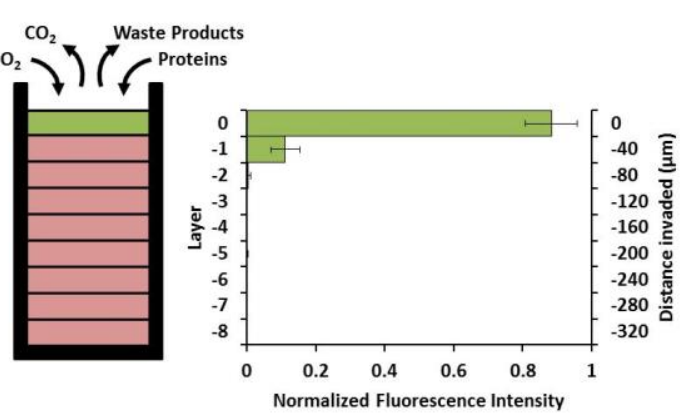

C

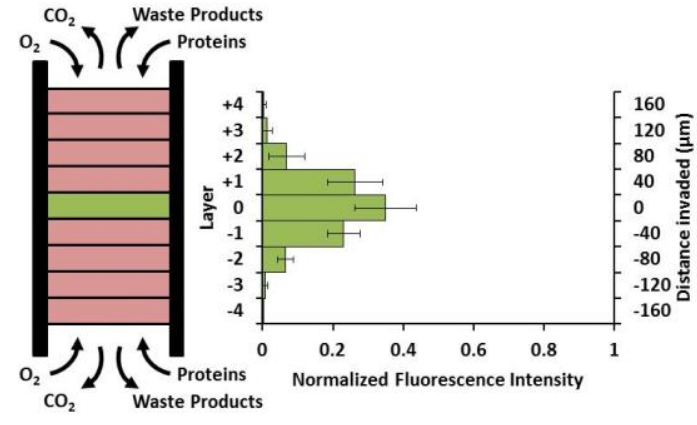

B

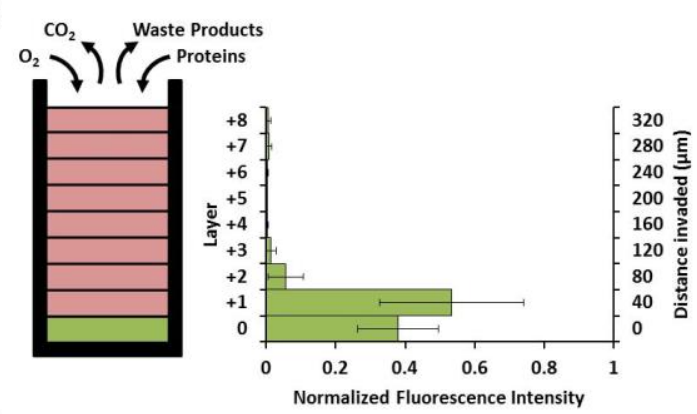

D

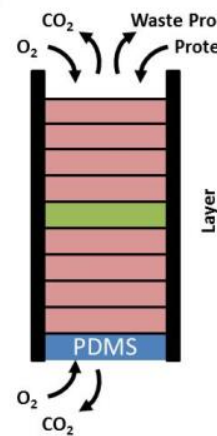

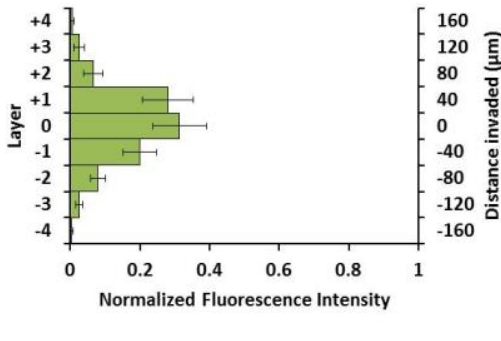

Figure 4. Oxygen is the primary chemoattractant within the invasion stack for A549-HGF-M cells. A-D) Schematic of the invasion stack with the seeded layer shown in green (positioned at the top, middle, or bottom of the stack), layers of matrigel shown in pink, layer of PDMS shown in blue (permeable to oxygen but not nutrients), and acrylic holder shown in black (impermeable to oxygen and nutrients). A) Cells were positioned at the top of the stack and nutrients and oxygen were available only from the top of the stack. B) Cells were positioned at the bottom of the stack and nutrients and oxygen were available only from the top of the stack. C) Cells were positioned in the middle of the stack and nutrients and oxygen were available at both the top and bottom of the stack. F) Cells were positioned in the middle of the stack, nutrients were available only from the top of the stack, and oxygen was available from both the top and bottom of the stack. Error bars represent the standard deviation for 20 replicate zones. 

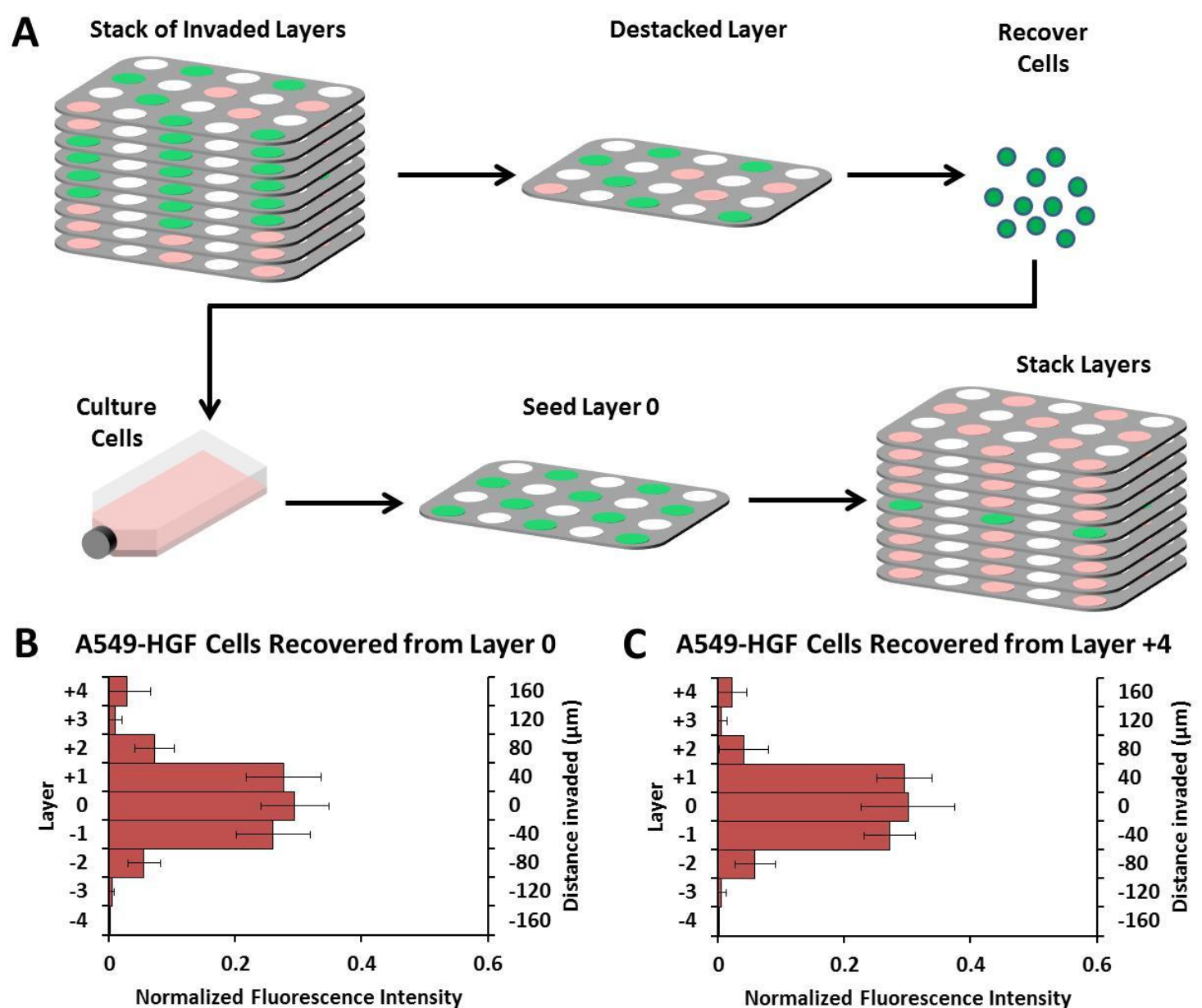

C A549-HGF Cells Recovered from Layer +4

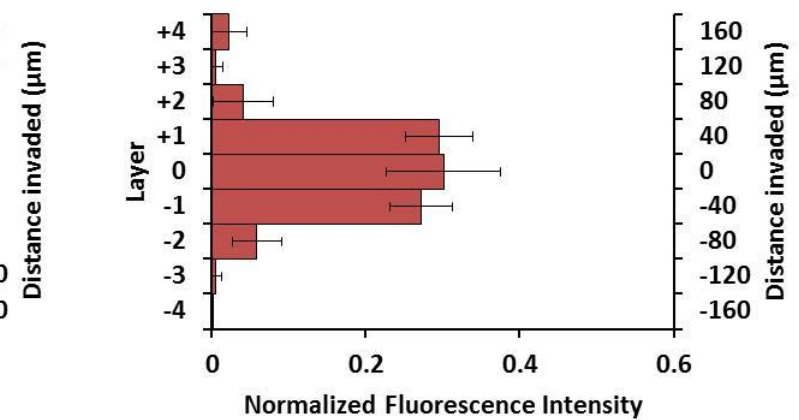

Figure 5. Local environment dictates distribution of invading cells. A) Schematic of generating a sub-line of cells recovered from a given layer of a stack and reimplanted into the invasion assay. B-C) Distribution of A549-HGF cells recovered from layer 0 (B) and layer +4 (C) of the same invasion stack that was cultured for 24 hours with 100,000 cells/zone in the seeded layer. Error bars represent the standard deviation for 20 replicate zones. 


\section{A Paper-Based Invasion Assay: Assessing Chemotaxis of Cancer Cells in Gradients of Oxygen}

Bobak Mosadegh $^{\mathrm{a}, \mathrm{b} \dagger} *$, Matthew R. Lockett ${ }^{\mathrm{a}}$, Kyaw Thu Minn $^{\mathrm{a}}$, Karen A. Simon ${ }^{\mathrm{a}}$, Karl Gilbert ${ }^{\mathrm{a}}$, Shawn Hillier ${ }^{\mathrm{c}}$, David Newsome ${ }^{\mathrm{c}}$, Howard Li ${ }^{\mathrm{c}}$, Amy Hall ${ }^{\mathrm{c}}$, Diane M. Boucher ${ }^{\mathrm{c}}$, Brenda K. Eustace $^{\mathrm{c} *}$ and George M. Whitesides ${ }^{\mathrm{a}, \mathrm{b}} *$

${ }^{a}$ Department of Chemistry and Chemical Biology, Harvard University

12 Oxford Street, Cambridge, MA 02138

${ }^{\mathrm{b}}$ Wyss Institute of Biologically Inspired Engineering, Harvard University

60 Oxford Street, Cambridge, MA 02138

${ }^{c}$ Vertex Pharmaceuticals Incorporated

50 Northern Avenue, Boston, MA 02210

*Authors to whom correspondence should be addressed.

Email: gwhitesides@gmwgroup.harvard.edu

Email: brenda_eustace@vrtx.com

Email: bmosadegh@gmwgroup.harvard.edu

${ }^{\dagger}$ Authors contributed equally 


\section{Materials and Methods}

Cells and Culture Reagents. We purchased A549 cells from the American Type Culture Collection. A549 cells engineered to constitutively express hepatocyte growth factor (HGF) were transduced with a HGF retrovirus using polybrene $(8 \mu \mathrm{g} / \mathrm{mL})$, and clones prepared from single cells selected with neomycin. The retroviral stock, a pLNCX2 plasmid (neomycin selection) expressing human HGF under the CMV promoter were harvested from 293T cell supernatants. A second retroviral stock, a pLNCX2 plasmid (puromycin selection) expressing luciferase under the CMV promoter was also harvested from 293T cells. We confirmed the clones expressed HGF with an ELISA assay (Quantikine, R\&D Systems). A high expressing clone was transduced with the luciferase retrovirus and population selected with puromycin. Luciferase expression was ascertained using SteadyGlo (Promega). All cells were cultured at $37{ }^{\circ} \mathrm{C}$ and $5 \%$ $\mathrm{CO}_{2}$ in DMEM medium containing $10 \%$ fetal bovine serum and $1 \%$ PenStrep.

Preparation of Paper Layers. We used a Xerox ColorQube 8870 printer to print wax patterns onto one side of a sheet of Whatman 105 lens paper. The layers of paper were then placed on a layer of aluminum foil, and baked at $150{ }^{\circ} \mathrm{C}$ for $10-15$ seconds; this short baking period allowed the wax to melt throughout the thickness of the paper. We cut the wax-patterned scaffolds from the layer of lens paper with a Versa Laser-Universal Laser VL-300 laser cutter. Each scaffold contained 20 hydrophilic zones surrounded by hydrophobic wax (Fig. 1). Each scaffold was sterilized in a bath of ethanol for approximately $30 \mathrm{~min}$ and then air-dried in a laminar flow hood under UV irradiation.

Preparation of the Invasion Stacks. Cultures of A549 cells were maintained in tissue culture flasks. Prior to preparing the invasion assay, we: i) detached the cells from the tissue culture 
flask (Trypsin-EDTA, 5 minute incubation at $37^{\circ} \mathrm{C}$ ), ii) washed the cells in the appropriate, serum-containing medium, iii) pelleted the cells (1000 x $g$, five minutes) and removed the medium, and iv) resuspended the cells in Matrigel to a final concentration of $10^{8}$ cells $/ \mathrm{mL}$ (the cells and Matrigel were kept on ice to prevent gelation). We next pipetted the suspension of cells directly onto the hydrophilic zones of the layer of paper $(1 \mu \mathrm{L} /$ zone for a final concentration of 100,000 cells/zone), and placed the "seeded layers" in a petri dish of pre-warmed culture medium $\left(37^{\circ} \mathrm{C}\right)$. For each seeded layer, we prepared eight "invaded layers" by pipetting $1 \mu \mathrm{L}$ of Matrigel/zone. The seeded and invaded layers contained the same 20-zone pattern, which consisted of 10 zones in which we added either Matrigel (invaded layers) or cells suspended in Matrigel (the seeded layer). The remaining 10 zones were "blank" and contained neither cells nor Matrigel. The blank zones were used to account for autofluorescence of each layer of paper, and background fluorescence arising from the staining protocols described below.

Prior to assembling the invasion stacks, each layer was placed in a petri dish containing DMEM medium and cultured overnight at $37{ }^{\circ} \mathrm{C}$ in an environment of $5 \% \mathrm{CO}_{2}$. The seeded layers and the invaded layers were incubated in separate petri dishes. The layers of paper were stacked in an acrylic holder (Fig. 1), which was machined specifically for the layers of lens paper. The seeded layer was placed in the middle of the invasion stack, with an equal number of invaded layers above and below the seeded layer. Once assembled in the acrylic holder, the invasion assay was placed in DMEM medium and cultured at $37{ }^{\circ} \mathrm{C}$ and in an environment of $5 \%$ $\mathrm{CO}_{2}$ for a desired period of time; the stack was then disassembled and each layer of paper analyzed (protocols described below in detail). 
Analysis of the Invasion Stacks. We analyzed the cells in each layer of paper of the destacked invasion stack with fluorescence images (Typhoon FLA 9000 laser scanner, GE) or confocal microscopy (Zeiss LSM 710 upright microscope).

Viability staining protocol. After destacking, each layer of the invasion stack was placed in a 6 well plate containing Dulbecco's phosphate-buffered saline (DPBS). The layers were washed two times with DPBS, and then fluorescently stained with a solution of calcein-AM (Invitrogen; $0.4 \mu \mathrm{g} / \mathrm{mL}$ in DPBS). We incubated the layers in the calcein solution for 20 minutes at $37{ }^{\circ} \mathrm{C}$ in an environment of $5 \% \mathrm{CO}_{2}$, and then washed each layer with DPBS twice before imaging in the Typhoon laser scanner: $488 \mathrm{~nm}$ excitation/band pass blue 1 filter $(530 \mathrm{~nm}), 50$-micron resolution images.

Recovery Protocol. To recover the cells from a layer of lens paper we placed each layer in a $50 \mathrm{~mL}$ Falcon tube containing $5 \mathrm{~mL}$ of Accumax (Innovative Cell Technologies) and incubated the solution at $37^{\circ} \mathrm{C}$ for 30 minutes on a shake table. The layers of paper were removed from the tubes, and the cells were washed with DMEM medium, centrifuged at 1,000 rpm for 5 minutes, and re-suspended with media to the desired concentration (e.g., 1,000 cells $/ \mu \mathrm{L}$ ).

To determine the percentage of cells recovered from the above process, we first stained the cells will calcein-AM prior to recovery, and compared the fluorescence intensity of each zone of a layer of paper before and after recovery. To determine the viability of the recovered cells, we plated approximately 500 cells in a each well of a 6-well plate, and determined the number of live cells after a 2-, 3-, 4-, and 5-day incubation with a CellTiter-Glo Luminescent Cell Viability Assay (Promega) according to the manufacturer's directions. 
Inoculation of Mice with Tumor Cell Lines. All animal studies were approved by the Institutional Animal Care and Use Committee in accordance with the guidelines set forth by the U.S. Public Health Service Policy on Humane Care and Use of Laboratory Animals. Mice were housed under standard conditions in approved facilities with 12 hour light/dark cycles and given food and water ad libitum. Male athymic Nu/Nu nude mice were purchased from Charles River Laboratories. Prior to inoculation, A549, A549-HGF, or A549-HGF-M cells were resuspended at $2 \times 10^{7}$ cells $/ \mathrm{ml}$ in a 1:1 mixture of phosphate buffered saline:Matrigel (BD Biosciences). All three cell lines expressed GFP. Each mouse was injected in the right flank with $0.1 \mathrm{ml}$ of the cell suspension.

Assessment of Metastasis in vivo. On Day 22 (Fig. S2), post inoculation of tumor cells, some mice $(n=10)$ were euthanized by asphyxiation with carbon dioxide and the number of lung metastases quantified using a Leica MZ16F Stereomicroscope with a green fluorescent protein filter. The lungs were excised and placed under the microscope where the number of metastases in a single plane of the lung was quantified by eye. An additional cohort of mice $(n=12-15)$ were euthanized when the tumor volume reached approximately $1,000 \mathrm{~mm}^{3}$, at which time the number of lung metastases was also enumerated. Tumor volumes were measured with calipers twice per week. 


\section{Supplemental Figures:}

A

B

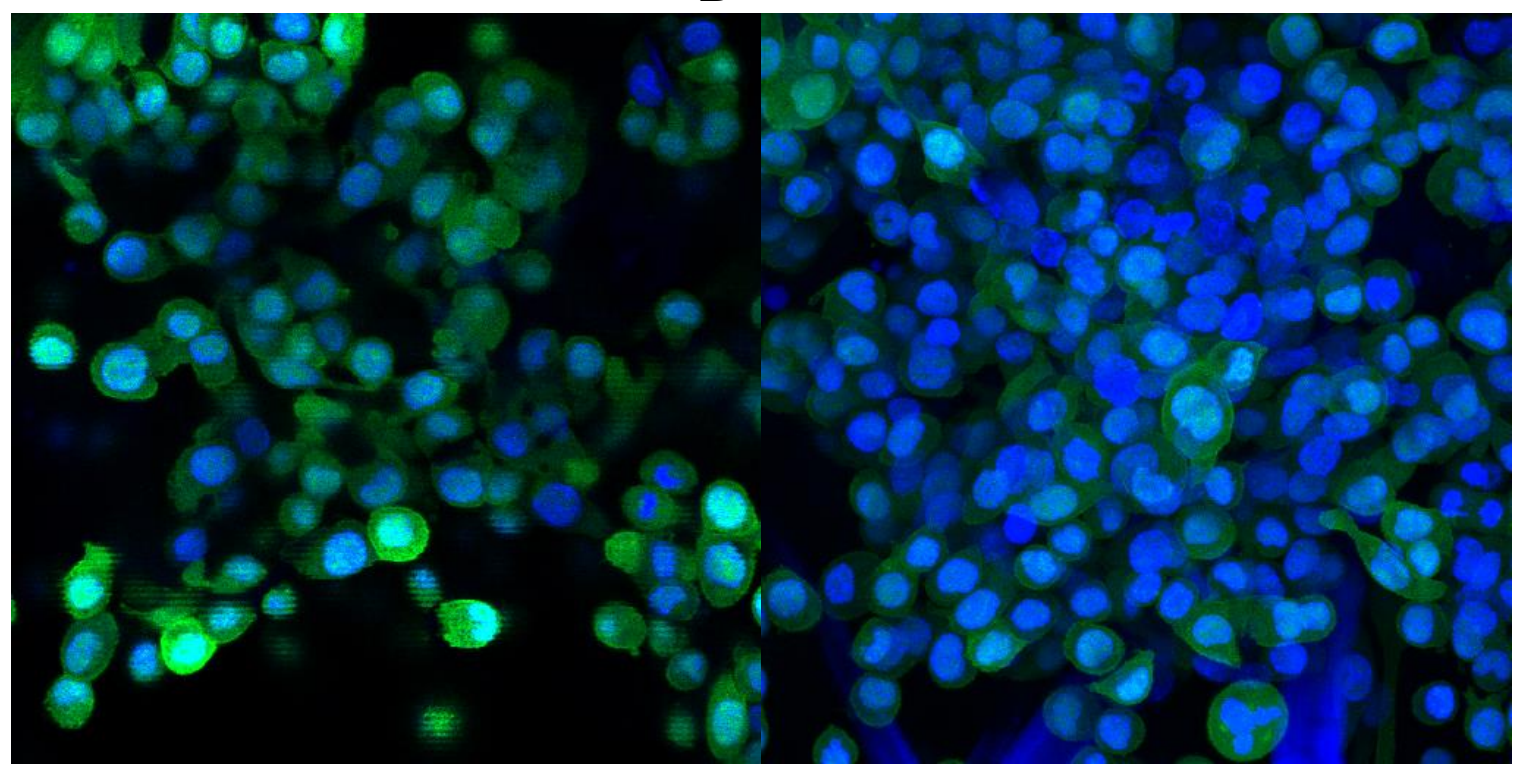

Supplemental Figure 1. A-B) A549-HGF-M cellsed in layer +4 after 24 hours within the invasion stack. All cells expressed GFP. (A) Single confocal scan of layer +4. (B) Maximum intensity projection of total thickness of layer +4 . Green is GFP, Blue is DAPI. 

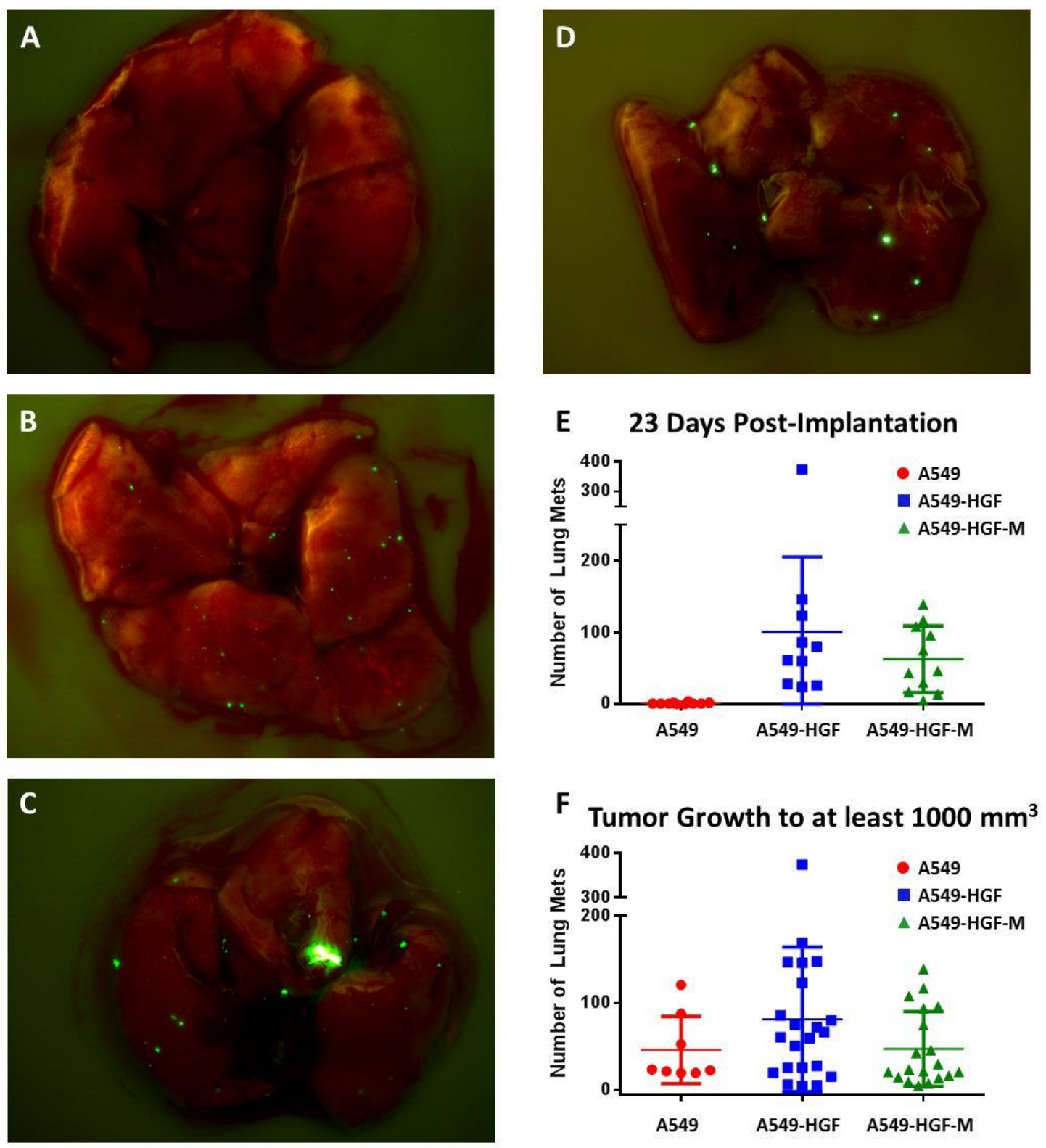

Supplemental Figure 2. A-C) Image of a mouse lung, 23 days post-implantation with A549 (A), A549-HGF (B), and A549-HGF-M (C) cells implanted subcutaneously on the hind flank of the mouse. All three cell lines expressed GFP. D) Image of mouse lung when the A549 xenograft tumor reached 1,000 $\mathrm{mm}^{3}$. E) Plot of number of lung metastases 23 days post-implantation of A549, A549-HGF, and A549-HGF-M cells. F) Plot of number of metastases to the lung when the primary tumor reached at least $1000 \mathrm{~mm}^{3}$ for A549, A549-HGF, and A549-HGF-M cells. 